The following manuscript, titled Earthquakes within Earthquakes: Patterns in Rupture Complexity, is a preprint submitted to EarthArXiv and has not been peer reviewed

\title{
Earthquakes within Earthquakes: Patterns in Rupture Complexity
}

\author{
Philippe Danré*1,2, Jiuxun Yin ${ }^{1}$, Bradley P. Lipovsky ${ }^{1}$, and Marine A. Denolle ${ }^{1}$ \\ ${ }^{1}$ Earth and Planetary Sciences \\ Harvard University, Cambridge, MA, USA \\ ${ }^{*}$ To whom correspondence should be addressed; E-mail: philippe.danre@ens.fr \\ ${ }^{2}$ Département de Géosciences \\ École Normale Supérieure, PSL Research University, Paris, France
}

\begin{abstract}
Earthquake source time functions carry information about the complexity of seismic rupture. We explore databases of source time functions of earthquakes and find that source time functions are composed of distinct peaks that we call subevents. We observe that earthquake complexity, as represented by the number of subevents, grows with earthquake magnitude. We find that subevent magnitudes are nearly proportional to their corresponding main event magnitude. We show that the main event magnitude can be estimated after observing only the first few subevents.
\end{abstract}

1 line summary Pattern in the complexity of earthquake source time function suggests a self organization in ruptures.

Whether earthquake complexity contains underlying structure is fundamental to the issue of earthquake predictability. Recent work has suggested that such structure permits a sense of determinism whereby observations made in a short period of time at the beginning of an earthquake may be used to infer the overall event size and style (1-3). Previous studies have found that early P waves arrivals inform the overall earthquake size $(1,4)$, but 7 these correlations bear large uncertainties that necessitate additional information, such as attenuation, for meaning8 ful early magnitude estimate (5-7). The first P wave arrival, however, represents only a small fraction of potentially 
The following manuscript, titled Earthquakes within Earthquakes: Patterns in Rupture Complexity, is a preprint submitted to EarthArXiv and has not been peer reviewed

deterministic information (8).

Earthquake seismic signals are the convolution of the source time function (STF), or spatially-averaged slip rate history, and the Green's function, which represents the effects of wave propagation from the earthquake fault to the seismometer (9). The basic structure of the STF is an initial acceleration of seismic moment followed its deceleration. When averaged over many events, STFs reach a maximum value around $30-50 \%$ of their duration $(2,10,11)$, which suggests a "weak determinism" whereby the moment of the earthquake is at least twice that released when the moment-rate decreases. Although this trend describes large ensembles of events, STFs exhibit significant variation between events as well as complexity within a single event (11). Here, we take advantage of existing STF catalogues ( $(12,13)$; see Supplementary Materials) to explore this complexity.

We describe STFs as a sum of "subevents," or time-domain Gaussian pulses (14). We refer to our iterative fitting procedure as a subevent decomposition (Supplementary Materials). Examples of such decomposition are shown in Figure 11. The subevent decomposition tunes the duration and amplitude of each subevent to better represent the catalogued STF. We consider only subevents that are greater than $10 \%$ of the STF maximum value and longer than $1 \mathrm{~s}$ in duration. We refer to each subevent as having a duration and moment, keeping in mind the inherent non-uniqueness of the STF due to its spatially averaged character.

Our main result is that subevent moment $M_{S}$ scales with the total moment $M_{0}$ (Figure 2 a) according to $\log _{10} M_{S}=0.8 \log _{10}\left(M_{0}\right)+3.06$. This scaling implies that subevents do not have an intrinsic length scale, but instead have uniform scaling with total event moment. We note several caveats of this analysis. First, a lower bound on subevent moment is determined by our detection threshold. We find, however, that the scaling relation is not sensitive to the choice of threshold (see Supplementary Materials). Second, the amplitude of the scaling could be biased toward lower values because we ignore the smallest subevents that have a duration less than 1 second. Thus, we believe that the scaling is between 0.8 and 1 . The data quality being limited to signals of duration longer than $1 \mathrm{~s}$, we retain the threshold of 0.1 and the implication of an artificial lower bound in subevent size. Additionally, the use of a symmetric Gaussian function for the pulse shape may not be not physical (15), but the reliable frequency content of the database limits us to use smooth functions. 
The following manuscript, titled Earthquakes within Earthquakes: Patterns in Rupture Complexity, is a preprint submitted to EarthArXiv and has not been peer reviewed

Using the number of subevents as a measure of earthquake complexity, we find our second main result: that earthquake complexity scales with earthquake size (Figure 3 3 ). In general, M5.5 earthquakes are constituted of about 1-4 subevents, while the M8+ earthquakes are composed of 8-10 subevents. This trend is clearer than that found by $(16)$ who used the zero crossings in the time derivative of STFs in two databases $(10,16)$ to quantify complexity. The growth in the number of observed subevents is more pronounced for the magnitudes greater than M6.75, which source dimension of $\sim 15 \mathrm{~km}$ corresponds to the seismogenic depth of crustal earthquakes. The M9.0 2011 Tohoku Earthquake is the outlier of the SCARDEC dataset, with only few subevents given its size. The Tohoku earthquake is well documented for being a single and large slip-patch $(17,18)$ hence bears a low complexity index in our analysis. Strike slip earthquakes tend to have greater complexity than other modes (see Supplementary Materials), perhaps due to their complex multi-fault geometry (Kokoxili $2001(19,20)$, Denali 2002 (21), Sumatra 2012 (22,23), Kaikoura 2016 (24)). Strike slip event complexity may be over estimated, however, by contamination of the direct seismic phases with their reverberation in the water column (25).

These two main results are self-consistent. Note, for example, that ratio between seismic moments of a M8 and an M6 is about 1000, yet the M8 has about only 5 additional subevents. Thus, it is not possible for a M8 earthquake to be composed of subevents of the same size as the ones found in a M6 earthquake. Instead, the observations imply that the subevent size grows with the main event size.

To better understand our observations, we turn to earthquake dynamic rupture simulations. Subevents occur naturally in these simulations when incorporating a self-similar fault pre-stress distribution (power-law exponent of 0.8 , a random phase (26-28)). Friction is described using the linear slip weakening model on a two-dimensional fault loaded out of the plane. The fault is $200 \mathrm{~km}$ long and the nucleation length is $1.6269 \mathrm{~km}(29)$. By randomly selecting a nucleation location, we construct a suite of 600 events to reproduce a spread in event magnitude of three orders of magnitudes. We reconstruct the STF by integrating the function that described the rate of moment per unit of fault width over the fault dimension (see Figure 1). However, the simulated STF contain a high frequency content (about $20 \mathrm{~Hz}$ ) whereas the observed SCARDEC STFs are only reliable up to $1 \mathrm{~Hz}$ due to data limitation. We thus smooth the simulated moment-rate with a Gaussian filter of width $2 \mathrm{~s}$. 
The following manuscript, titled Earthquakes within Earthquakes: Patterns in Rupture Complexity, is a preprint submitted to EarthArXiv and has not been peer reviewed

We perform the same subevent decomposition for both observed and modeled STFs. We find similar patterns between our modeled and observed STFs (Figure $2 \mathrm{p}$ and $3 \mathrm{p}$ ). We find greater variability in the number of subevents observed than modeled, which can be due in part to modeling strategies (such as a lower degree of structural and/or fault property heterogeneity than reality) or to the over-simplified Green's function used in SCARDEC. Nevertheless, in both observed and simulated sets of STF, the number of subevents in general grows with earthquake size (Fig. 2).

Earthquake Early Warning (EEW) is a natural application of the scaling between subevent and main event size. Specifically, our results suggest that the final magnitude may plausibly be estimated by observing only a small number of initial subevents. To test this idea, we extract an STF constructed from the September 28, 2018 M7.5 Palu Earthquake in Indonesia from the USGS database ( (30), accessed on 19/10/2018, Figure 4). This event was not used in forming the main scaling relation. We begin scanning the STF at time $t=0$ and we fit the first subevent at $t=1.8 \mathrm{~s}$. At this time we estimate the subevent moment and duration by fitting a Gaussian, and then apply the above-described scaling relation to estimate the total event moment.

We find that our algorithm predicts a magnitude of M7.2 at $1.8 \mathrm{~s}$ of rupture time, while only M6.3 equivalent of moment magnitude was released, and while the final size of the event is M7.5 (Fig. (4). Upon the second subevent detection at $3.6 \mathrm{~s}$, which we constrain as being no smaller than $15 \%$ of the amplitude of the first peak, we calculate an updated moment magnitude estimate and take the mean of both estimates. We iterate as the rupture evolves and refine the magnitude estimates based on the distributions of the previous individual estimates by taking the mean value. This allows us to stabilize the magnitude estimates as the variability in subevent size will fluctuate the estimates. An alternative approach to rapid moment estimation based on observing the maximum moment rate (11) would require approximately an additional $10 \mathrm{~s}$ to our approach. This calculation ignores latency in wave propagation and EEW systems (31).

We have performed a similar exercise as in Figure 4 for the entire SCARDEC database and find that we can predict the final magnitude with an uncertainty of 0.27 in moment magnitude within the first $20 \%$ of rupture duration (see Supplementary Materials). We find that the largest subevent is detected within 30-50\% of the rupture 
The following manuscript, titled Earthquakes within Earthquakes: Patterns in Rupture Complexity, is a preprint submitted to EarthArXiv and has not been peer reviewed

time, which is related to the slight asymmetrical shape found by $(2,10,11)$. Nevertheless, these biases can be accounted for in a future improvement and application of this scaling.

Our work is mainly limited by the globally averaged attenuation model and the simplicity in the Green's function used to reconstruct the source time function from the teleseismic seismograms. Because we are limited to seismic waves with frequencies lower than $1 \mathrm{~Hz}$, improved considerations of 3D Green's functions and in particular in attenuation models will enhance the data set at lower magnitudes. Improved methodology might also better inform whether fault heterogeneity can be constrained by subevent distributions. But overall, our work suggests that earthquake ruptures, seen through their complexity, are self organized and have a sense of deterministism, and that patterns in their complexity may be explained by physical models.

\section{References and Notes}

1. E. L. Olson, R. M. Allen, Nature 438, 212 (2005).

2. D. Melgar, G. P. Hayes, Geophysical Research Letters 44, 9691 (2017).

3. D. E. Goldberg, D. Melgar, Y. Bock, R. M. Allen, Journal of Geophysical Research: Solid Earth 0 (2018).

4. R. M. Allen, H. Kanamori, Science 300, 786 (2003).

5. Y.-M. Wu, H.-Y.Yen, L. Zhao, B.-S. Huang, W.-T. Liang, Geophysical Research Letters 33 (2006).

6. S. Colombelli, A. Zollo, G. Festa, M. Picozzi, Nature Communications 5, 3958 EP (2014).

7. S. Noda, W. L. Ellsworth, Geophysical Research Letters 43, 9053 (2016).

8. M. Vallée, et al., Science 358, 1164 (2017).

9. K. Aki, Journal of Geophysical Research 72, 1217 (1967).

10. Y. Tanioka, L. J. Ruff, Seismological Research Letters 68, 386 (1997).

11. M.-A. Meier, J. P. Ampuero, T. H. Heaton, Science 357, 1277 (2017). 
The following manuscript, titled Earthquakes within Earthquakes: Patterns in Rupture Complexity, is a preprint submitted to EarthArXiv and has not been peer reviewed

12. M. Vallée, V. Douet, Physics of the Earth and Planetary Interiors 257, 149 (2016).

13. G. P. Hayes, Earth and Planetary Science Letters 468, 94 (2017).

14. Z. Zhan, H. Kanamori, V. C. Tsai, D. V. Helmberger, S. Wei, Earth and Planetary Science Letters $\mathbf{3 8 5}, 89$ (2014).

15. E. Tinti, E. Fukuyama, A. Piatanesi, M. Cocco, Bulletin of the Seismological Society of America 95, 1211 (2005).

16. H. Houston, Journal of Geophysical Research: Solid Earth 106, 11137 (2001).

17. S. Ide, A. Baltay, G. C. Beroza, Science 332, 1426 (2011).

18. G. Shao, C. Ji, D. Zhao, Geophysical Research Letters 38 (2011).

19. Y. Klinger, et al., Bulletin of the Seismological Society of America 95, 1970 (2005).

20. A. Tocheport, L. Rivera, J. V. der Woerd, Bulletin of the Seismological Society of America 96, 1729 (2006).

21. D. Eberhart-Phillips, et al., Science 300, 1113 (2003).

22. H. Yue, T. Lay, K. D. Koper, Nature 490, 245 (2012).

23. L. Meng, et al., Science 337, 724 (2012).

24. Y.-Y. Wen, K.-F. Ma, B. Fry, Bulletin of the Seismological Society of America (2018).

25. H. Yue, J. Castellanos, C. Yu, L. Meng, Z. Zhan, Geophysical Research Letters 44, 9573 (2017).

26. P. M. Mai, G. C. Beroza, Journal of Geophysical Research: Solid Earth 107, ESE (2002).

27. J. Ripperger, J.-P. Ampuero, P. M. Mai, D. Giardini, Journal of Geophysical Research: Solid Earth 112 (2007).

28. T. Candela, et al., Journal of Geophysical Research: Solid Earth 117 (2012).

29. K. Uenishi, J. R. Rice, Journal of Geophysical Research: Solid Earth 108, 2042 (2003). 
The following manuscript, titled Earthquakes within Earthquakes: Patterns in Rupture Complexity, is a preprint submitted to EarthArXiv and has not been peer reviewed

125 30. U. S. G. Survey .

126 31. S. E. Minson, M.-A. Meier, A. S. Baltay, T. C. Hanks, E. S. Cochran, Science Advances 4 (2018). 
The following manuscript, titled Earthquakes within Earthquakes: Patterns in Rupture Complexity, is a preprint submitted to EarthArXiv and has not been peer reviewed
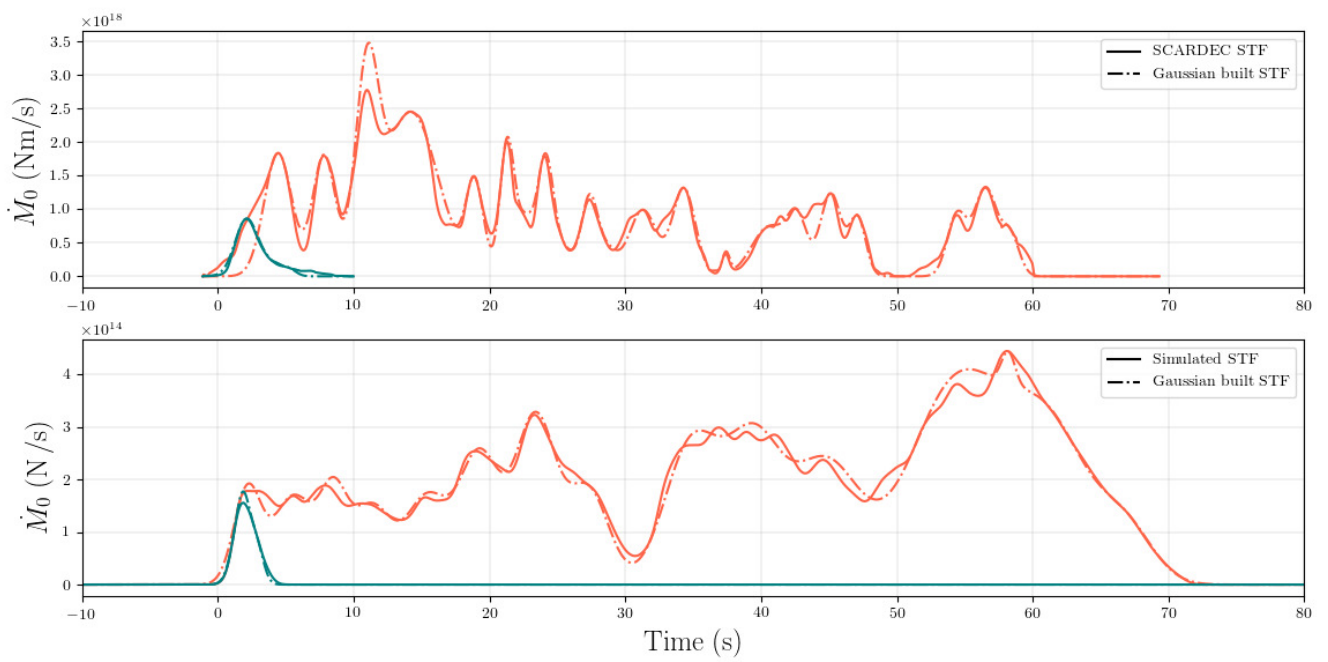

Figure 1: Complexity in observed and modeled STF. (Top) Two observed STFs from the SCARDEC database from the 2015/07/03 M6.2 Leyte Earthquake in green and from the 1994/03/14 M7.1 Central Mid-Atlantic Ridge Earthquake in orange. (Bottom) Two modeled STF in moment-rate per unit of fault width for a large (orange) and small (green) events. In both figures, solid lines show the original SCARDEC STF and dashed lines show to the STF reconstructed with the Gaussian subevent functions. In both modeled and observed cases, the larger event exhibits more and bigger subevents than that of the small event.

127

\section{Acknowledgements}

Funding: This research was supported by the Southern California Earthquake Center (SCEC) award \#17001, under National Science Foundation Cooperative Agreement EAR-1033462 and US Geological Survey Cooperative Agreement G12AC20038. The SCEC contribution number of this paper is 8978. Author contributions: P.D. designed the subevent decomposition, performed the observations, analysis, made the figures, and wrote the original draft. J.Y. performed the simulation analysis and helped editing the manuscript. B.P.L. advised the simulations part and helped with the manuscript. M.D designed and managed the project, served an advisory role to J.Y. and P.D, and edited manuscript and figures. None of the authors have any conflict or competing interest. The SCARDEC database is open to the public http://scardec.projects.sismo.ipgp.fr/and the SBIEMLAB codes are maintained by JP Ampuero at https://github.com/jpampuero/semlab. The python and matlab scripts are available in supplementary materials. 
The following manuscript, titled Earthquakes within Earthquakes: Patterns in Rupture Complexity, is a preprint submitted to EarthArXiv and has not been peer reviewed
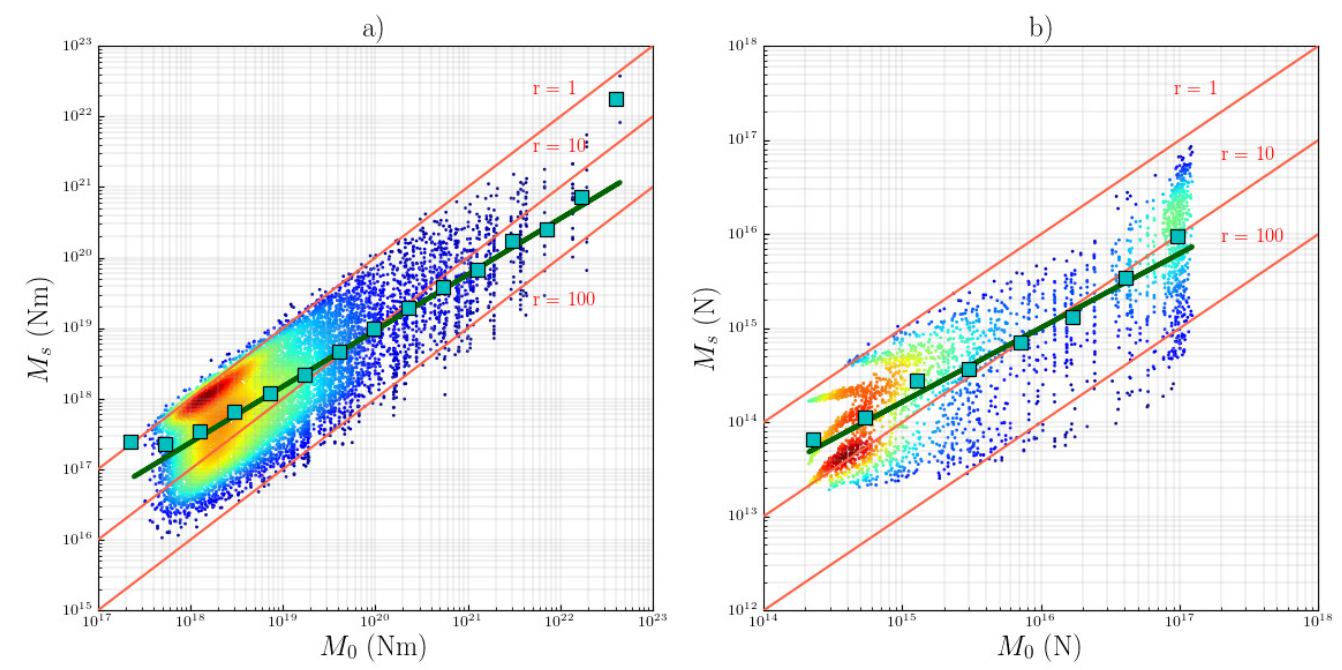

Figure 2: Scaling of subevent moment with earthquake size: individual subevent moments against main earthquake moment for observed (a) and modeled (b) STFs. In both figures, dots represent a single measurement, squares represent the medians over moment bins. Red lines indicate a ratio of seismic moment of subevent to earthquake $r$ of 1, 10, and 100 respectively. The dots are colored according to their probability density. The green solid thick line is a linear regression performed over the the log-log distribution of individual measurements, $M_{S} \propto M_{0}^{0.8}$ for the observations and for the simulations, which we later use for rapid magnitude estimates (see Figure 4).

\section{Supplementary materials}

Materials and Methods

Supplementary Text

Figs. S1 to S7

Tables S1

Movie S1

References (32-41) 
The following manuscript, titled Earthquakes within Earthquakes: Patterns in Rupture Complexity, is a preprint submitted to EarthArXiv and has not been peer reviewed
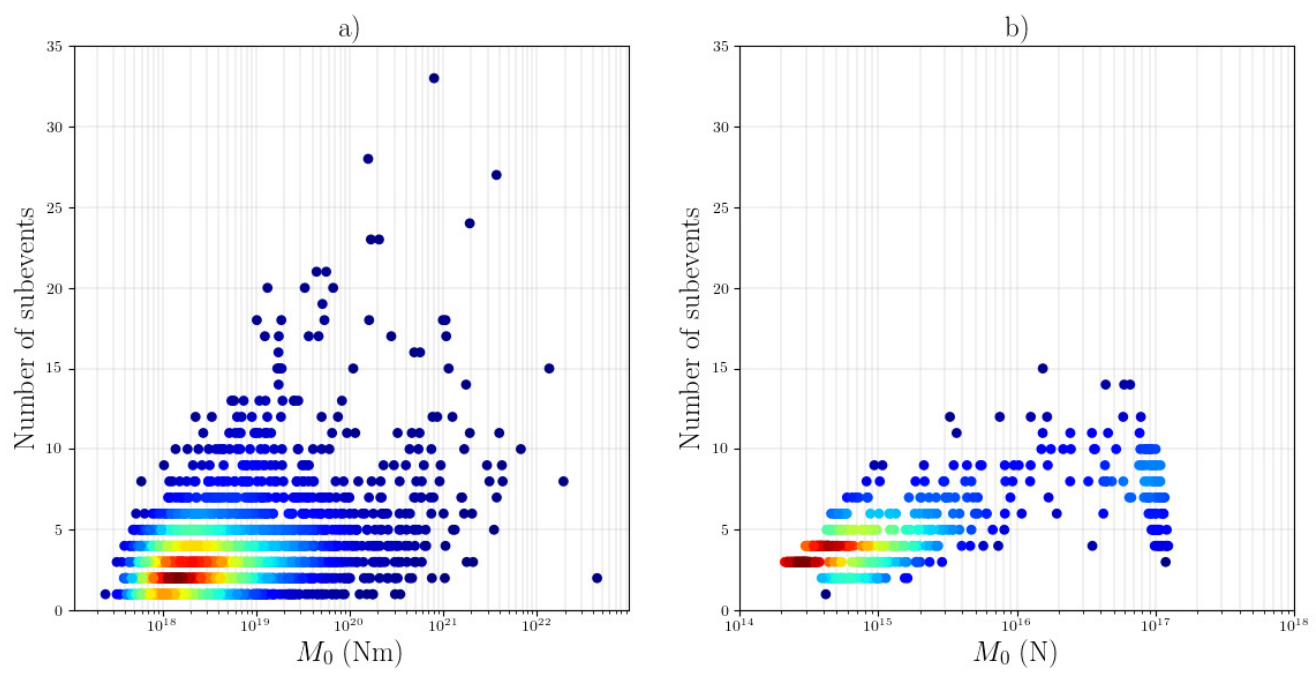

Figure 3: Earthquake complexity grows with earthquake size: Number of subevents as a function of seismic moment of the main event for (a) the observed STFs and (b) the modeled STFs. Dots are the individual earthquakes, orange squares are median in moment bins. Dots are colored according to the probability density function. The growth in complexity is monotonic with earthquake size for the observations, except for the M9.0 2011 Tohoku earthquake, and the growth is noticeable for the simulations. 
The following manuscript, titled Earthquakes within Earthquakes: Patterns in Rupture Complexity, is a preprint submitted to EarthArXiv and has not been peer reviewed
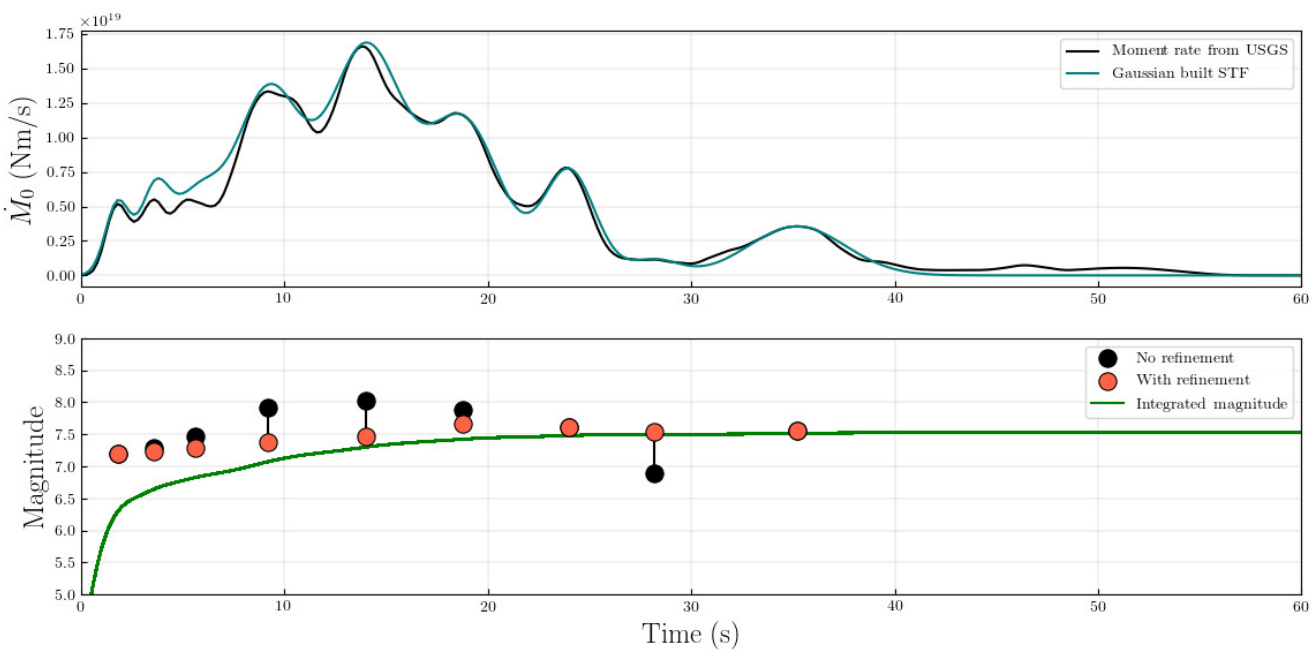

Figure 4: Magnitude prediction in the recent 2018 M7.5 Palu Earthquake, Indonesia. (Top) USGS Source time function estimate (https://earthquake.usgs.gov/archive/product/finite-fault/ us 1000h3p4/us/1539813424642/moment_rate.mr, last accessed on 10/19/2018) in black, and our Gaussian-built STF in green dash line. (Bottom) "Real time" magnitude estimate throughout the rupture: green curve is the magnitude difference from the cumulative seismic moment, black dots are the magnitude estimates from individual subevents fitting (using scaling statistics from Figure 3), red dots are the median of all previous magnitude estimates. See Supplementary Materials for the detailed algorithm. 


\section{Materials and Methods}

\section{Source time functions databases.}

Our results use the SCARDEC database of source time functions http://scardec.projects.sismo.ipgp.fr/, last accessed on 11/11/2018. The database contains over 3,000 events of magnitudes 5.5 and greater from 1992 to 2017. The database is well established whereby earthquake source parameters have been compared with other published catalogs $(12,32$ 36). The SCARDEC dataset of STF, as published on the website, are constructed from global P phases $(\mathrm{P}, \mathrm{pP}, \mathrm{sP}, \mathrm{PP}, \mathrm{PcP})$ whereby wave propagation and attenuation are compensated with simple Green's function calculated in radially symmetric global Earth model. Because attenuation is better constrained for frequencies lower than $1 \mathrm{~Hz}$, we do not interpret signals that are shorter than $1 \mathrm{~s}$ in the following. The sampling rate in the database is $0.0703 \mathrm{~s}$.

To validate our results, we use the United State Geological Survey (USGS) STF database that contains 180 events (13, to date of 12/4/2018). The STF from this dataset are constructed from kinematic inversion for slip based on the $\mathrm{Ji}$ et al. (2002) (37) algorithm that is a non-linear inversion that solves for rupture velocity, slip, and slip rate. The waveforms used in the fit are P, $\mathrm{S}$, and surface waves (13). This database has also been validated for various earthquake-focus publications, is the official data product provided by the USGS, and commonly serves as a baseline for source time function and finite fault model comparison. The STFs in the USGS database have variable sampling rates. Figure S1 shows the same analysis as in the main manuscript and it serves as a validation with an independent database.

\section{Gaussian decomposition algorithm}

In the following, we elaborate on the Gaussian decomposition algorithm. It is similar in spirit as in Zhan et al., (2014) (14). We perform the decomposition forward in time. The algorithm starts from time zero:

a. Go forward in time and detect an amplitude peak $\mathrm{S}_{\mathrm{S}}$ (local maximum over 3 points) at time ts that satisfies $\mathrm{S}_{\mathrm{S}}>0.1 \max (\mathrm{STF})$

b. Fit a Gaussian function centered around ts the STF by setting its amplitude to $\mathrm{S}_{\mathrm{S}}$ and by varying its width with a grid search over the RMS width $\sigma$.

c. Substract to the STF the fitted Gaussian function (some residual peaks may be made detectable.

d. Go forward and back to step a.

If the detection duration is less than $1 \mathrm{~s}$, we ignore the peak. Otherwise, the detection is considered a subevent and its moment $\mathrm{M}_{\mathrm{S}}$ is the value of the Gaussian function integrated over time.

The choice of a threshold is necessary to ignore spurious residuals that are not resolvable by the data (high frequency or low amplitudes). We tried different thresholds $(0.01,0.05,0.10,0.15)$ as shown Figure S2 for the 0.01 threshold. The spread in subevent moment is large for this low threshold, whereby smaller subevents can be detected. However, if the spread of subevent moment changes, the scaling of subevent vs main event moment does not change. Due to the limitations 
in the SCARDEC data set at high frequency, we choose a threshold of 0.1 to only retain the main subevents.

\section{Application to Earthquake Early Warning (EEW).}

In a real-time application of our findings, we do not have prior knowledge of the maximum value of the STF, which is known a posteriori. Thus, we modify the detection algorithm, and show one example in main manuscript Figure 4. In order to avoid fitting residuals, we proceed as follow:

1) Detect a first peak:

a) Compute its moment ${ }^{1} \mathrm{M}_{\mathrm{s}}$ and duration ${ }^{1} \mathrm{~T}_{\mathrm{s}}$.

b) Use the scaling relation to get ${ }^{1} \mathrm{M}_{0}$ from ${ }^{1} \mathrm{M}_{\mathrm{S}}$ and combine with magnitude-moment relation: ${ }^{1} \mathrm{M}_{\mathrm{W}}=0.8 \log _{10}\left({ }^{1} \mathrm{M}_{\mathrm{S}}\right)-9.3$

2) Detect the following peak "i" if it has at least 0.15 times the amplitude of the first peak (for the Palu Earthquake application to EEW) or with the $10 \%$ of the maximum STF criteria when applied to the whole SCARDEC database:

a) Compute its moment ${ }^{i} \mathrm{Ms}$ and duration ${ }^{\mathrm{i}} \mathrm{T}_{\mathrm{s}}$.

b) Use the scaling relation to get ${ }^{i} \mathrm{M}_{0}$ from ${ }^{i} \mathrm{M}_{\mathrm{s}}$ and combine with magnitude-moment relation: ${ }^{\mathrm{i}} \mathrm{M}_{\mathrm{W}}=0.8 \log _{10}\left({ }^{1} \mathrm{M}_{\mathrm{S}}\right)-9.3$

c) Take the median of the individual magnitude estimates ${ }^{i} \mathrm{M}_{\mathrm{W}}=\operatorname{median}\left({ }^{(1->i)} \mathrm{M}_{\mathrm{W}}\right)$.

3) Go back to 2 .

This is the method implemented for Figure 4 of main manuscript. We apply this to all events in SCARDEC database. We show the difference between the magnitude ground truth and the predicted value against rupture time, which we normalize to the final source duration. We take the definition of duration similar to (33) whereby the duration is measured until the STF amplitude becomes lower than $10 \%$ the peak of the STF, to avoid long tail that cannot be resolved due to unmodeled near-source scattering.

In Figure S3, each dot is a magnitude estimate at the peak time of a subevent ${ }^{i} \mathrm{M}_{\mathrm{W}}-\mathrm{M}_{\text {true }}$. The color of the dot is chosen to illustrate the power density function of the dots (blue is few events, red is a large number of events). First estimates can come in as early as $5 \%$ of the source duration with an underprediction of about 0.5 magnitude increment. Between 30-50\%, our method overpredicts the magnitude by an increment of 0.5 . This is because the largest subevent tends to be in the central part of the STF (11). This is also visible in Figure 4. After $50 \%$ of the source duration, the uncertainty in magnitude estimates is 0.27 in magnitude estimate.

\section{Properties of the subevents}

To verify that the subevents have physically consistent values of duration $T_{S}$ and moment magnitude $\mathrm{M}_{\mathrm{S}}$, we estimate their stress drop as if they were small independent quakes and compare them to the main event stress drop. Note that subevents are not independent, but rather dynamically connected through fracture mechanics. Following $(34,38,39)$, the model-dependent stress drop is:

$$
\Delta \sigma=\frac{7}{16} *\left(M /(k \beta)^{3}\right) \text { where } f c=0.6 / T, \mathrm{k}=0.32, \text { and } \beta=3900 \mathrm{~m} / \mathrm{s}
$$


The duration $\mathrm{T}$ is equal to the width where the Gaussian STF is at least $10 \%$ of the maximum value for the subevents, and as the time between zero and the last time where the STF reaches $10 \%$ of the maximum STF amplitude for the main event. We compute the stress drop for all subevents, their main event and take their ratio.

We show these estimates in Figure S4. We find a median value of $8 \mathrm{MPa}$ for the subevents stress drop, whereas the median value of these calculated for the main events is $0.3 \mathrm{MPa}$, a low and model-dependent value similar to the findings of (34). The median ratio of subevent stress drop to main event stress drop is 19 . One interpretation is that these peaks in moment-rate are events of larger stress drop than the rest of the event.

As mentioned in main text, complexity of earthquake as a count of subevents is stronger for strike slip than for dip slip earthquakes (Figure S5). The number of subevents for events with a dip higher than $70^{\circ}$ (mostly strike slip) is higher than for those of lower dip (normal and thrust), especially at high magnitudes. This might argue for the need of statistics like in Figure 3 but made for subsets of main events, depending on their type, to add precision in the statistics performed.

\section{Numerical simulations of dynamic ruptures.}

Our simulations are based on 2D mode III dynamic crack model, efficiently solved by the spectral boundary integral methods (SBIEMLAB, code developed by Jean-Paul Ampuero, http://web.gps.caltech.edu/ ampuero/software.html). The ingredients for our simulations are listed below:

a) Basic parameters in the simulations

Model domain is $400 \mathrm{~km}$ long, with $200 \mathrm{~km}$ long fault domain and $200 \mathrm{~km}$ boundary domain, and is uniformly discretized by 4096 grids with grid size $\Delta x=97.7 \mathrm{~m}$. Other basic parameters are listed in the Table S1.

b) Constitutive relation

We choose the basic linear slip weakening law to prescribe the friction on the fault:

$$
\mu= \begin{cases}\frac{\mu_{d}-\mu_{s}}{D_{C}} s+\mu_{s} & s \leq D_{C} \\ \mu_{d} & s>D_{C}\end{cases}
$$

where $\mathrm{s}$ is the slip, $\mu_{d}$ and $\mu_{s}$ are the dynamic and static friction coefficients, respectively. Dc is the critical slip distance, we choose $D_{C}=0.8 \mathrm{~m}$ in the main text but also test $D_{C}=0.4 \mathrm{~m}$ and $1.2 \mathrm{~m}$. The frictional parameters $\mu_{d}$ and $\mu_{s}$ are uniform on the fault in our model.

c) Heterogeneous pre-stress distribution

We produce a heterogeneous pre-stress distribution using the power spectral density PSD obeys a self-similar power law:

$$
\operatorname{PSD}(k)=k^{-\gamma},
$$

where $\mathrm{k}$ is the wavenumber, $\gamma$ is the Hurst exponents and we choose $\gamma=0.8$, which is constrained by the fault roughness observations (40). The phase of the pre-stress distribution 
is randomly generated from a uniform distribution in $\left[\begin{array}{ll}0 & 2 \pi\end{array}\right]$. Combining the PSD and random phases, we can produce a large population of statistically identical distributions.

Then we set the negative part of these distributions, normalize to the range between $\left[\mu_{d} \sigma_{0} \mu_{s} \sigma_{0}\right]$. To avoid the artifacts in STF from abrupt stopping of rupture at the fault boundary in the spectral boundary integral solutions, we further apply a Tukey taper on the pre-stress distributions (Fig.S6 (a)). To make sure the post-processing on the pre-stress distribution does not distort the original PSD, we also compare the spectra of unprocessed and processed pre-stress distributions in the frequency domain (Fig.S6 (b)). Within the length scale of the entire active fault zone $(200 \mathrm{~km})$, the self-similarity, that is, the slope of the pre-stress with post-processing is mostly preserved with slight distortion.

\section{d) $2 \mathrm{D}$ spontaneous dynamic rupture model (Mode III crack)}

To nucleate the spontaneous dynamic rupture, we randomly and artificially set an over-stressed nucleation area. We choose the region whose pre-stress peak is within $\mathrm{x}=[-3030] \mathrm{km}$ on the fault and nucleate rupture at that location. This setting helps to avoid the boundary effects of pre-stress distribution and thus ensure that most of the simulated events evolve on a stress condition that is statistically similar during the event. The nucleation length is based on the relation from (41):

$$
L_{C} \approx \frac{1.158 \mu D_{C}}{\left(\mu_{s}-\mu_{d}\right) \sigma_{0}} .
$$

For $D_{C}=0.8 \mathrm{~m}$, the corresponding $L_{C}=1626.9 \mathrm{~m} \approx 17 \Delta \mathrm{x}$, which also ensures the sufficient spatial resolution of our simulations. We set the size of nucleation zone to be $1.5 L_{C}=2440.4$ $\mathrm{m}$ and this is to guarantee the ending of quasi-static stage and beginning of dynamic unstable slip, after the nucleation (41).

For each $D_{C}$, we produce 600 pre-stress distributions that qualify the condition mentioned before, and finally get 600 simulated STFs with different event sizes.

\begin{tabular}{|l|l|}
\hline S wave velocity $\mathrm{V}_{\mathrm{S}}(\mathrm{km} / \mathrm{s})$ & 3.46 \\
\hline Density $\rho\left(\mathrm{kg} / \mathrm{m}^{3}\right)$ & 2,670 \\
\hline Shear modulus $\mu(\mathrm{GPa})$ & 32 \\
\hline Normal stress $\sigma_{0}(\mathrm{MPa})$ & 120 \\
\hline Dynamic friction $\mu_{d}$ & 0.525 \\
\hline Static friction $\mu_{S}$ & 0.677 \\
\hline$D_{C}(\mathrm{~m})$ & 0.8 \\
\hline
\end{tabular}


power law exponent $\gamma$

0.8

Table 1: properties of the simulations 

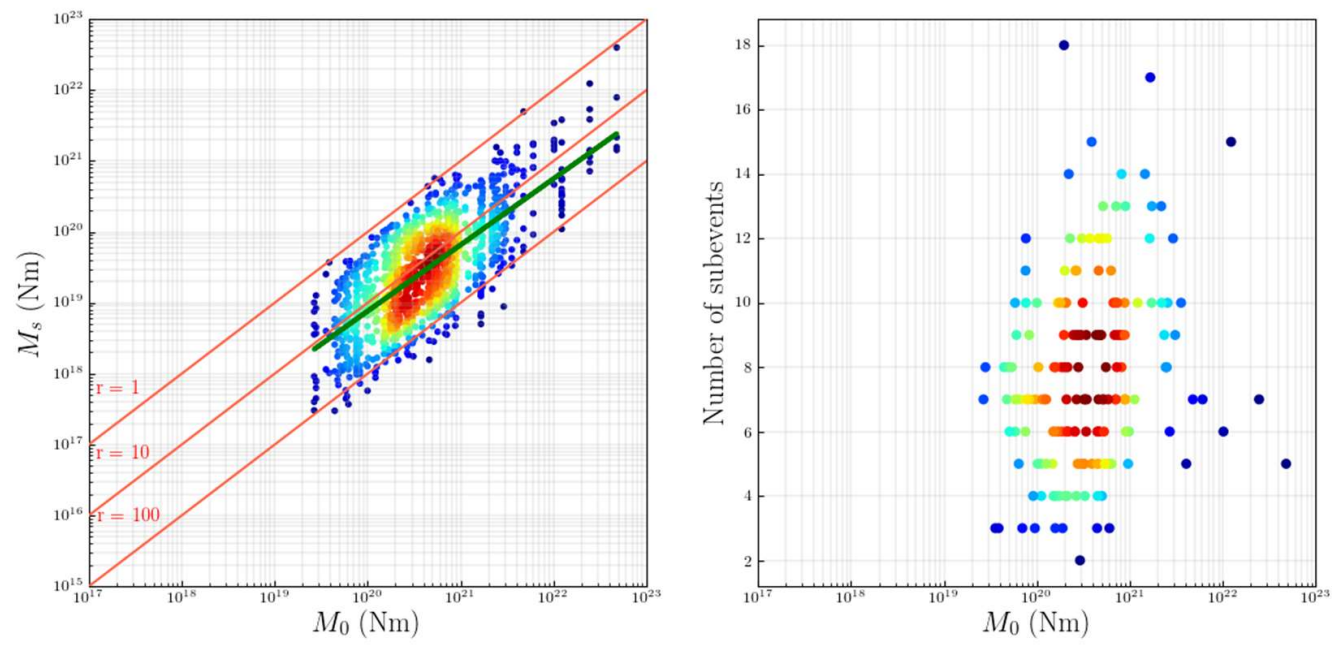

Figure S1. Results for the USGS STFs (Hayes, 2017). (a) Subevent moment Ms as a function of main event moment $\mathrm{M}_{0}$ for a peak detection with a $10 \%$ threshold. Green points are individual measurements of the moment, and squares are median results per 0.25 magnitude bins. The green line represents the result of the regression performed over the whole dataset. Finally, red lines represent the $\mathrm{r}=1,10$ and 100 ratios between the two moments. Despite a wider scatter, results still show a scaling similar to the SCARDEC results: $\mathrm{M}_{\mathbf{s}} \sim \mathrm{M}_{\mathbf{0}}{ }^{0.9}$ ( (b) Number of subevents as a function of main event moment, dots representing individual measurements, colored by their density distribution. There is an increase of complexity with main moment, less pronounced than for SCARDEC, maybe because of the lack of data. 


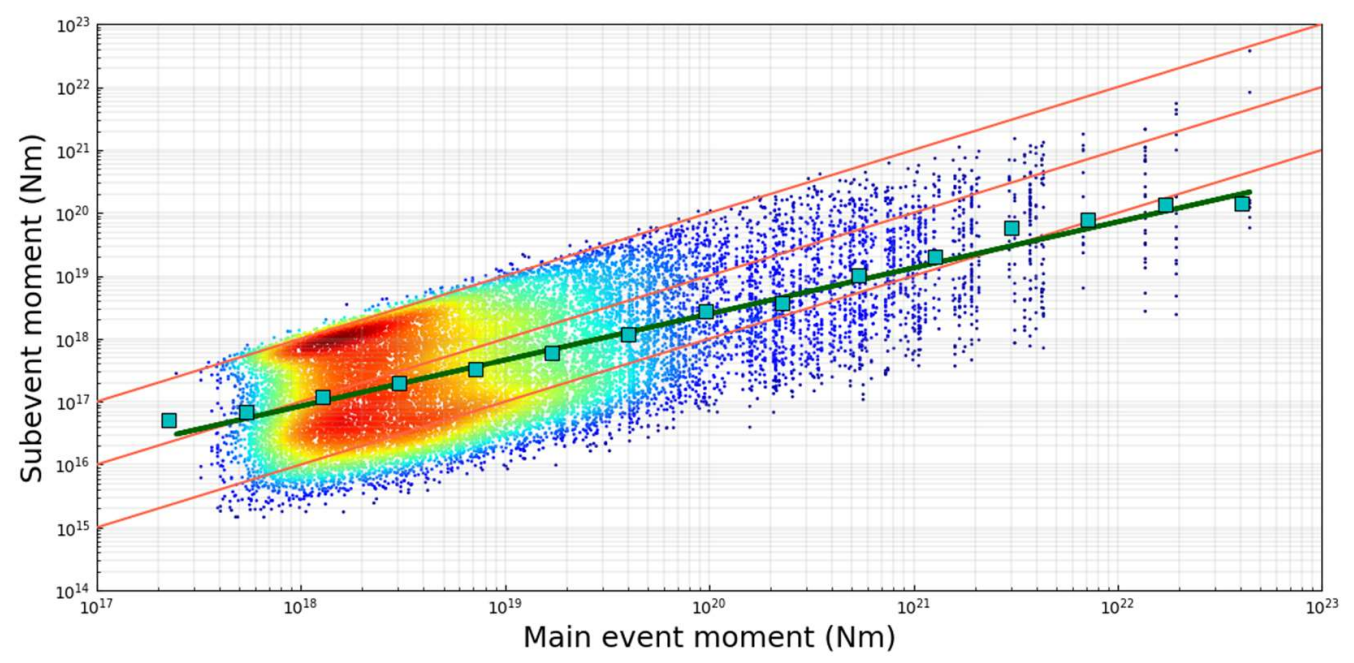

Figure S2. Subevent moment $\mathbf{M}_{\mathbf{S}}$ against main event $\mathbf{M}_{\mathbf{0}}$ : Similar to Figure 2 of main manuscript but with a 0.01 threshold. Dots are individual subevent measurements of given their main even moment. Dots are colored to highlight the probability density function (blue few elements, red many elements). Blue squares are median results per 0.25 magnitude bin, the error bar in the median of their bootstrapped median are smaller than the marker size. The green line is the linear regression performed over the whole dataset in a log-log space. Red lines represent the $r=1,10$, and 100 ratios moments. Despite a wider scatter, results still show a scaling similar to the 0.1 threshold results: $\mathrm{M}_{\mathrm{S}}=\mathrm{M}_{0}{ }^{0.8}$. It also shows that our detection becomes sensitive to the residuals from the Gaussian fitting, hence lower subevent moments. 


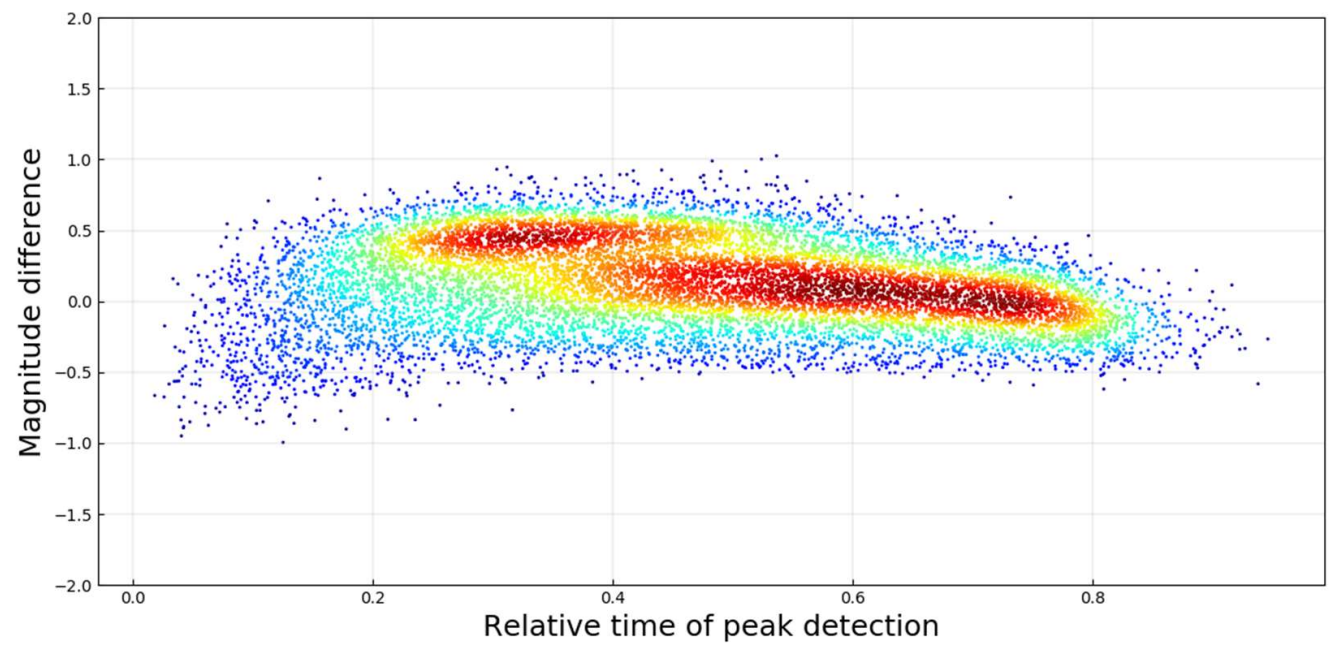

Figure S3. Magnitude estimates for all SCARDEC STFs. Similar to Figure 4 of main manuscript. Each dot is the difference between the SCARDEC moment magnitude and the one estimated from the moment scaling (Figure 3a) for each subevent of every event. For each earthquake, we proceed with the algorithm described in section 3. Between $20 \%$ and $40 \%$ of STF duration, our algorithm overpredicts the magnitude by 0.5 unit due to the fact that STFs tend to peak at that time, and thus experience the largest subevent. The estimates have an uncertainty of 0.27 magnitude before $20 \%$, of 0.31 magnitude between 20 and $50 \%$, and of 0.27 magnitude after $50 \%$. 


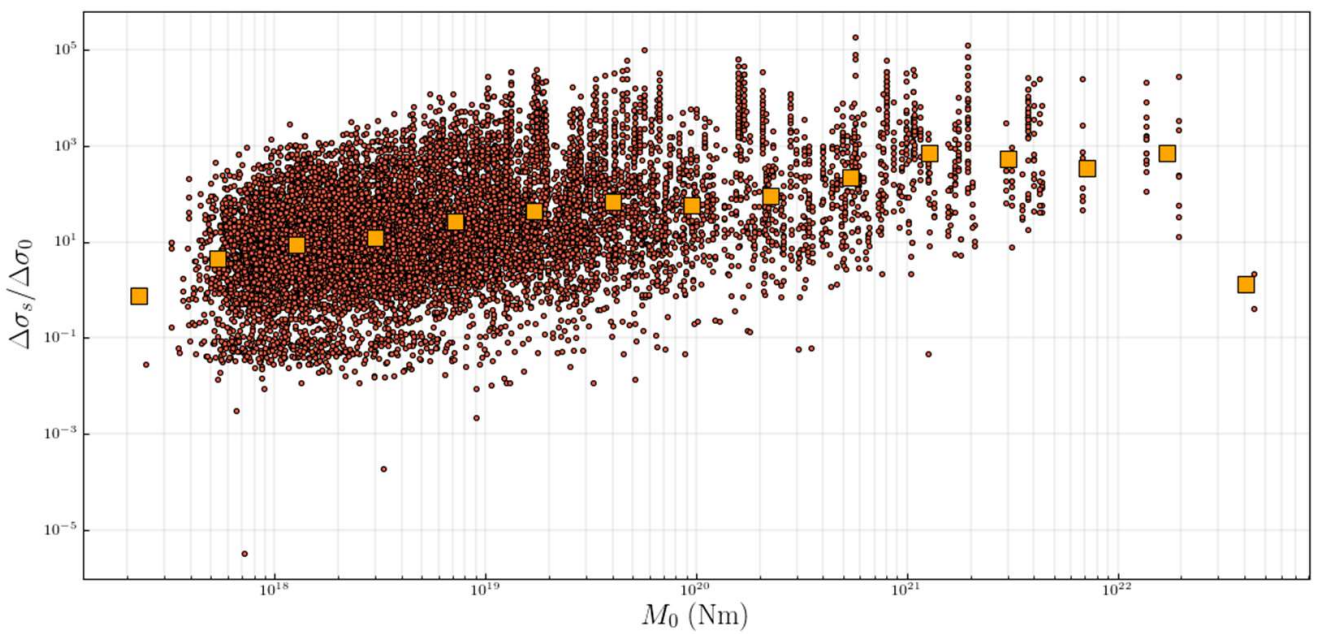

Figure S4. Ratio of subevent stress drop over main event stress drop. Each dot is an individual measurement of the ratio, squares represent the median ratio of 0.25 magnitude bins. The increase in the ratio is due to a growth of subevent stress drop instead of main event stress drop, as those exhibit a nearly constant stress drop around $0.3 \mathrm{MPa}$. 


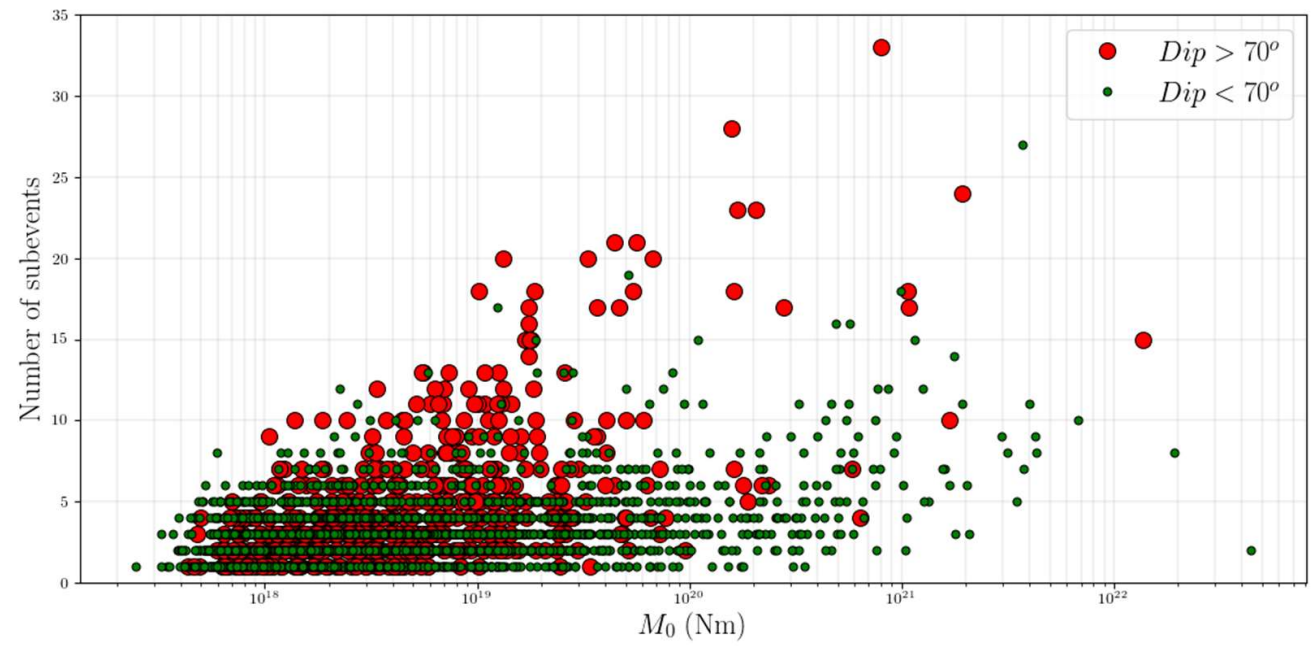

Figure S5. Number of subevents as a function of main event moment. We represent earthquakes with a dip higher than $70^{\circ}$ in red dots, and in green dots the results for main events with a dip lower than $70^{\circ}$. We are therefore able to see the influence of dip over rupture complexity, and this highlights the higher complexity for strike slip events. 

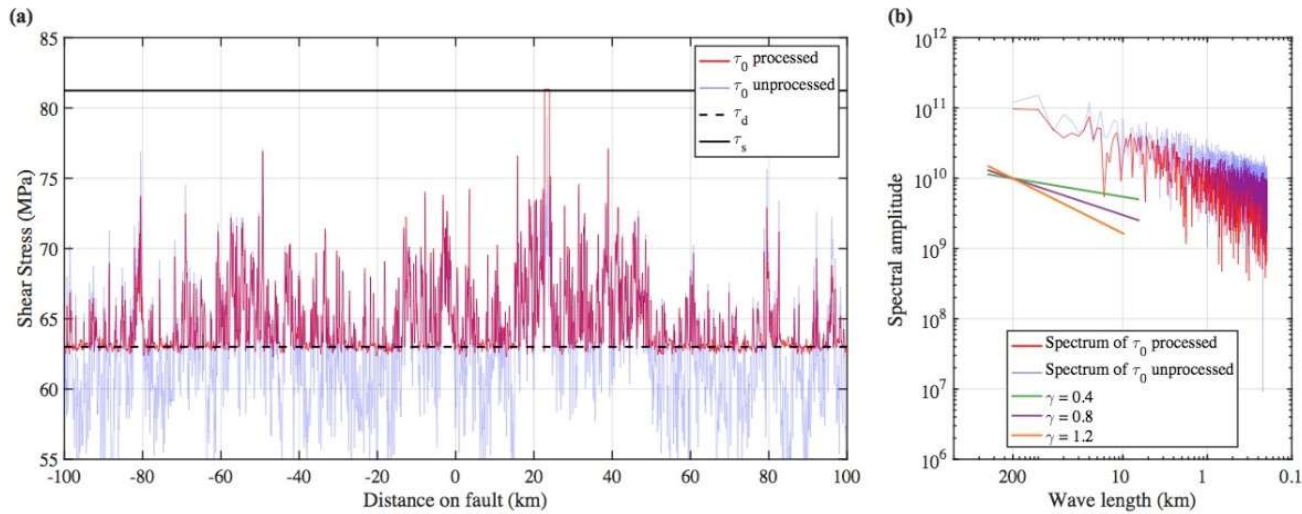

Figure S6: Pre-stress distribution and corresponding amplitude spectrum. (a) One example of the stochastically generated pre-stress distribution. Blue line shows the pre-stress distribution without any post-processing, while red line is the pre-stress after processing and used in the dynamic simulation. Black solid and dashed lines are the uniform fault strength and dynamic friction, respectively. (b) Comparison between the processed (red) and unprocessed (blue) prestress distributions in the frequency domain. Green, purple and orange lines show the references of different Hurst exponents of $0.4,0.8$ (used in this study) and 1.2, respectively. 
(a)

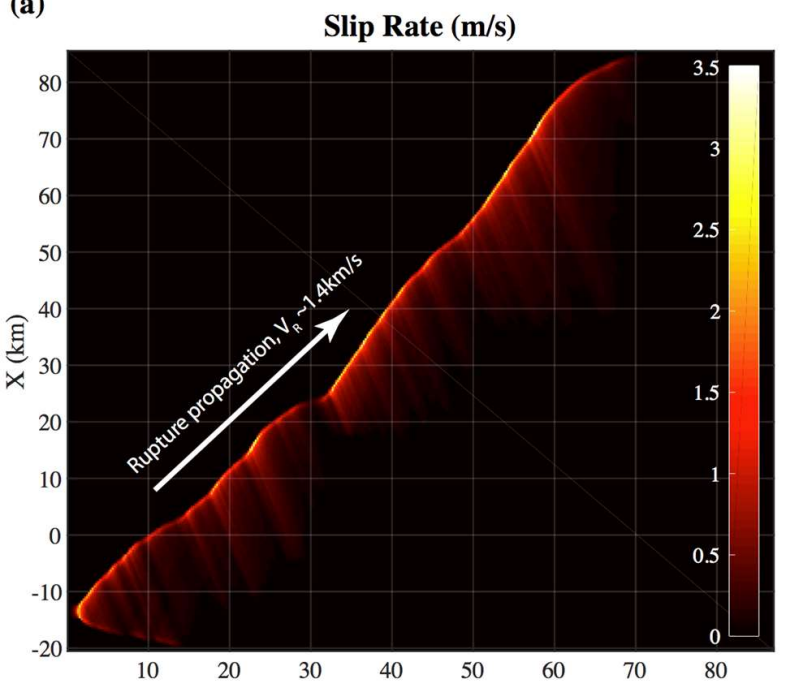

(e)

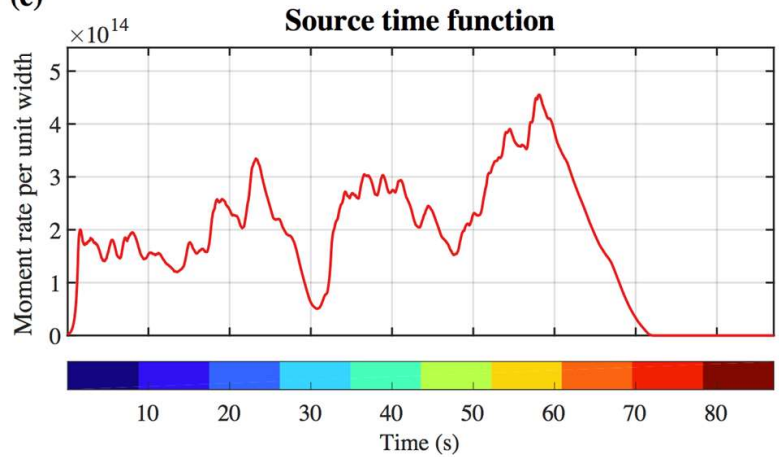

(b)

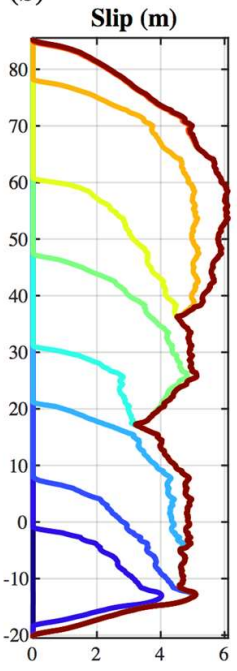

(c)

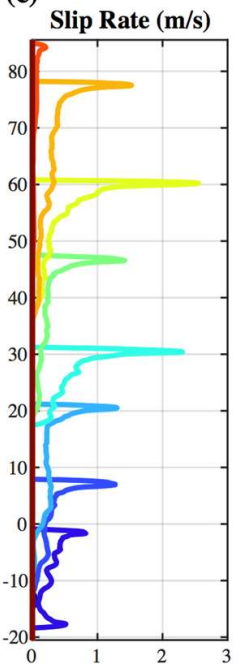

(d)

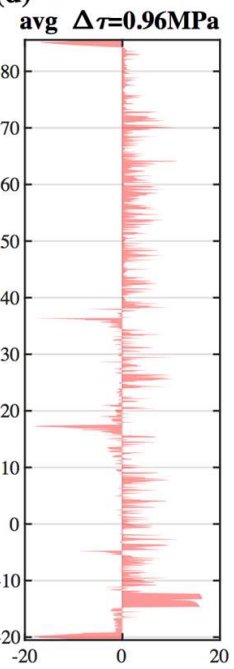

(f)

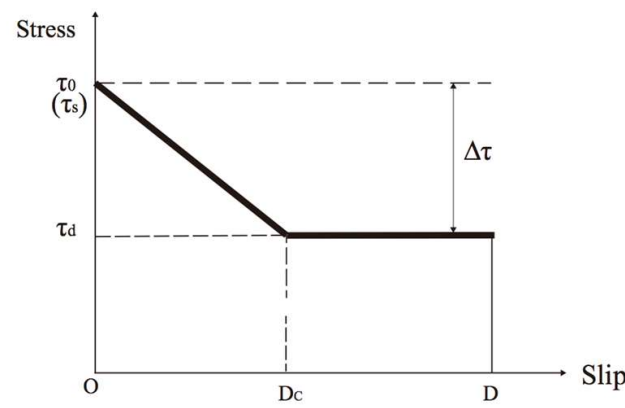

Figure S7: example of numerical simulation of the dynamic rupture. (a) shows the timespace distribution of slip rate, (b) the slip profile across the fault, (c) the slip rate as a function of time, (d) the stress change on the fault, (e) the final STF, (f) the linear slip weakening. 
Movie 1: Application of our results to the 09/28/2018 Palu Earthquake, Indonesia. We apply the Gaussian decomposition as detailed above, the algorithm described in section 3 to the STF of the Palu Earthquake, the final result being shown in Figure 4.

\section{References:}

32- M. Vallée, J. Charléty. , A. M. Ferreira, B. Delouis and J. Vergoz, SCARDEC: a new technique for the rapid determination of seismic moment magnitude, focal mechanism and source time functions for large earthquakes using body-wave deconvolution. Geophysical Journal International, 184: 338-358, 2011

33- M. Vallée, Source time function properties indicate a strain drop independent of earthquake depth and magnitude. Nature Communications, 4, 2013

34- F. Courboulex, M.Vallée, M. Causse, A. Chounet; Stress-Drop Variability of Shallow Earthquakes Extracted from a Global Database of Source Time Functions. Seismological Research Letters ; 87 (4): 912-918, 2016

35- M. Vallee and V. Douet. A new database of source time functions (stfs) extracted from the scardec method. Physics of the Earth and Planetary Interiors, 257:149 - 157, 2016

36- Chounet and M. Vallée, Global and interregion characterization of subduction interface earthquakes derived from source time functions properties. Journal of Geophysical Research: Solid Earth, 123, 5831-5852, 2018

37- Ji, D. J. Wald, and D. V. Helmberger. Source description of the 1999 hector mine, california, earthquake, part i: Wavelet domain inversion theory and resolution analysis. Bulletin of the Seismological Society of America, 92(4):1192-1207, 2002

38- Eshelby JD. The determination of the elastic field of an ellipsoidal inclusion, and related problems. Proc. R. Soc. Lond. A. 1957 Aug 20;241(1226):376-96.

39- Brune JN. Tectonic stress and the spectra of seismic shear waves from earthquakes. Journal of geophysical research. 1970 Sep 10;75(26):4997-5009.

40- T. Candela, F. Renard, Y. Klinger, K. Mair, J. Schmittbuhl, and E. E. Brodsky. Roughness of fault surfaces over nine decades of length scales. Journal of Geophysical Research: Solid Earth, 117(B8), 2012

41- Uenishi, K., \& Rice, J. R. Universal nucleation length for slip-weakening rupture instability under nonuniform fault loading. Journal of Geophysical Research: Solid Earth, 108(B1), 2003 